\title{
Synthesis and biological activity of some heterocyclic compounds containing benzimidazole and beta-lactam moiety
}

\author{
K F ANSARI and C LAL* \\ Department of Chemistry, Harcourt Butler Technological Institute, Nawabganj, Kanpur 208002 , \\ e-mail: c_lal12@rediffmail.com; kfahbti@gmail.com
}

MS received 22 January 2009; revised 7 August 2009; accepted 7 September 2009

\begin{abstract}
A number of $1 N$-substituted-2-methyl benzimidazole derivatives have been synthesized and tested for their antibacterial activities. The chemical structures of the newly synthesized compounds were verified on the basis of spectral and elemental methods of analyses. Investigation of antimicrobial activity of the compounds was done by disc diffusion method using Gram-positive ( $S$. aureus, $S$. mutans and $B$. subtilis), Gram-negative (E. coli, S. typhi and P. aeruginosa) bacteria and fungi (C. albicans, A. flavus and A. niger). Among the compounds tested $\mathbf{5 a}, \mathbf{5 b}, \mathbf{5 d}, \mathbf{5 i}, \mathbf{5} \mathbf{j}$ and $\mathbf{5 k}$ exhibited good antibacterial activities against Gram positive bacteria, while 5d and $\mathbf{5 i}$ also showed notable antifungal activity. Specially compounds $\mathbf{5 a}$ and 5b exhibited appreciable activity against $S$. aureus and B. subtilis comparable to reference drugs.
\end{abstract}

Keywords. 2-Methyl benzimidazole; azetidin-2-one; antimicrobial activity; ampicillin; nalidixic acid; amphotericin B.

\section{Introduction}

The benzimidazole ring is an important pharmacophore in modern drug discovery. A variety of benzimidazole are in use, like thiabendazole and flubendazole (anthelmintic), omeprazole and lansoprazole (antiulcerative) and astemizole (antihistaminic) (figure 1). The chemistry and pharmacology of benzimidazoles have been of great interest to medicinal chemistry, ${ }^{1,2}$ because its derivatives possessed various biological activities such as antioxidant, ${ }^{3,4}$ antimicrobial, ${ }^{5-10}$ antihelmintic, ${ }^{11-13}$ anticancer, ${ }^{14}$ antihypertensive, ${ }^{15}$ antineoplastic, ${ }^{16}$ anti-inflammatory, ${ }^{17,18}$ analgesic, ${ }^{19}$ antiprotozoal ${ }^{20,21}$ and anti-hepatitis $\mathrm{B}$ virus activity. ${ }^{22}$ In addition, a large number of antibiotics contain the 2-azetidinone (commonly known as $\beta$-lactam) moiety ${ }^{23}$ such as penicillin, cephalosporin and carbapenem (figure 2). It is also associated with a variety of therapeutic activities. ${ }^{24-28}$ In continuation of our work to develop potential antimicrobial molecules, ${ }^{29,30}$ we report here the synthesis of some derivatives of the title structure type containing the above mentioned moieties for evaluation of their antimicrobial activity.

*For correspondence

\section{Experimental}

All melting points were determined in an open capillary tube and are uncorrected. Infrared spectra were recorded in $\mathrm{KBr}$ on Perkin-Elmer RX1 spectrophotometer. The ${ }^{1} \mathrm{H}-\mathrm{NMR}$ and ${ }^{13} \mathrm{C}$-NMR spectra were measured in $\mathrm{CDCl}_{3}$ solutions on a Brucker DRX-300 $\mathrm{MHz}$ spectrometer using TMS as an internal reference (chemical shift in $\delta \mathrm{ppm}$ ). The mass spectra were recorded on a Jeol SX-102 instrument. Elemental analyses were carried out with Elementar Vario EL III elemental analyzer. Thin layer chromatography was performed on silica plates pre-coated with Merck Kiesegel 60 F254 and column chromatography with silica gel.

\subsection{Synthesis of 2-methyl-1H-benzimidazole-1- carbohydrazide (3)}

A mixture of compound $2(20 \mathrm{mmol})$ and hydrazine hydrate $(100 \% ; 20 \mathrm{mmol})$ in dry methanol $(40 \mathrm{ml})$ was refluxed on a steam bath for $4 \mathrm{~h}$ the excess solvent was removed under reduced pressure and the product crystallized from methanol to obtain compound 3. Yield $78 \%$; mp $173-175^{\circ} \mathrm{C}$; IR $(\mathrm{KBr})$ : $1632(-\mathrm{C}=\mathrm{N}), 1673(-\mathrm{C}=\mathrm{O}$ amido $), 3157\left(-\mathrm{NHNH}_{2}\right)$ $\mathrm{cm}^{-1} ;{ }^{1} \mathrm{H}-\mathrm{NMR}$ (DMSO- $\left.d_{6}, \delta \mathrm{ppm}\right): 2.58(s, 3 \mathrm{H}$, 


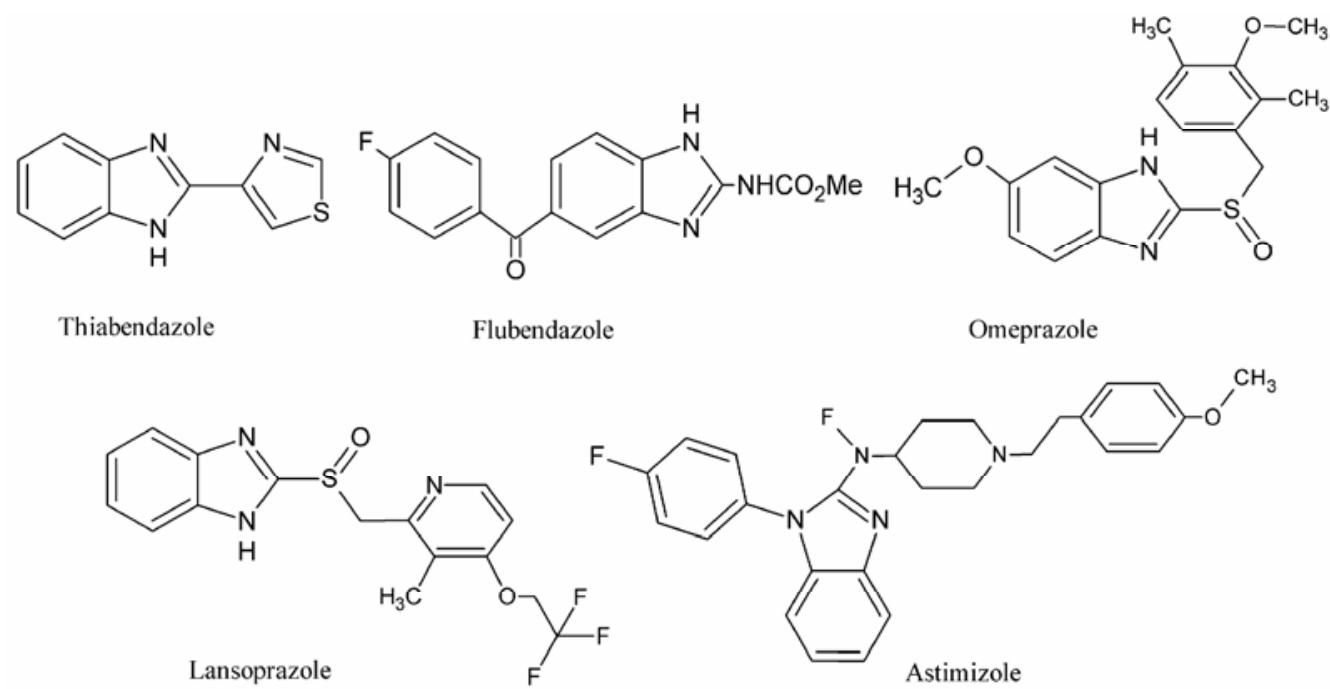

Figure 1. Clinically used benzimidazoles.<smiles>[R]C(=O)NC1C(=O)N2[C@@H]1SC(C)(C)[C@H]2C(=O)O</smiles>

Penicillin

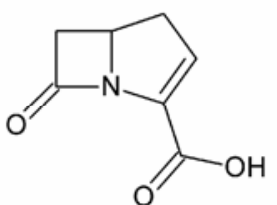

Carbapenem<smiles>[R]C(=O)N[C@@H]1C(=O)N2C(C(=O)O)=C([X])CSC12</smiles>

Cephalosporin

Figure 2. Antibiotics containing $\beta$-lactam ring.

$-\mathrm{CH}_{3}$ at $\mathrm{C}-2$ of benzimidazole), $2.60\left(s, 2 \mathrm{H},-\mathrm{NH}_{2}\right)$, 7.79-7.92 ( $m, 4 \mathrm{H}$, aromatic protons); EI-MS: 191 $\left(\mathrm{M}^{+}+1\right)$.

\subsection{2-Methyl-N'-[(substituted) alkyl/aryl} methylidene]-1-carbohydrazido-1H-benzimidazoles (4a-4m)

General procedure: To a stirred solution of compound 3 (30 mmol) containing 4-5 drops of glacial acetic acid in methanol $(30 \mathrm{ml})$ was added appropriate aldehyde $(30 \mathrm{mmol})$ and the mixture refluxed for $4-8 \mathrm{~h}$ on a steam bath. Methanol was removed under vacuum and the resultant semisolid treated with distilled water $(3 \times 10 \mathrm{ml})$. The separated solid was filtered, dried and recrystallized from ethanol to give title compounds.

2.2a $\quad N^{\prime}$-Ethylidene-1-carbohydrazido-2-methyl-1Hbenzimidazole (4a): IR (KBr): $1624(-\mathrm{C}=\mathrm{N}), 1588$ $(-\mathrm{N}=\mathrm{CH}), 1680 \quad(-\mathrm{C}=\mathrm{O}$ amido $), 3300 \quad(-\mathrm{C}-\mathrm{NH})$ $\mathrm{cm}^{-1} ;{ }^{1} \mathrm{H}-\mathrm{NMR}$ (DMSO- $\left.d_{6}, \delta \mathrm{ppm}\right): 1.34(d, 3 \mathrm{H}$,
$\left.-\mathrm{CHCH}_{3}\right), 2.48\left(s, 3 \mathrm{H},-\mathrm{CH}_{3}\right.$ at $\mathrm{C}-2$ of benzimidazole $), 3.95\left(q, 1 \mathrm{H},-\mathrm{CHCH}_{3}\right), 7.94-7.75(\mathrm{~m}, 4 \mathrm{H}$, aromatic protons); EI-MS: $217\left(\mathrm{M}^{+}+1\right)$.

$2.2 \mathrm{~b} \quad 2-$ Methyl- $N^{\prime}$-propylidene-1-carbohydrazidolH-benzimidazole (4b): IR ( $\mathrm{KBr}): 1657(-\mathrm{C}=\mathrm{N})$, $1605(-\mathrm{N}=\mathrm{CH}), 1681(-\mathrm{C}=\mathrm{O}$ amido $), 3336(-\mathrm{C}-\mathrm{NH})$ $\mathrm{cm}^{-1}$; ${ }^{1} \mathrm{H}-\mathrm{NMR}$ (DMSO- $\left.d_{6}, \delta \mathrm{ppm}\right): 1.00(t, 3 \mathrm{H}$, $\left.-\mathrm{CH}_{2} \underline{\mathrm{CH}}_{3}\right), 1.48\left(p, 2 \mathrm{H},-\underline{\mathrm{CH}}_{2} \mathrm{CH}_{3}\right), 2.35(s, 3 \mathrm{H}$, $-\mathrm{CH}_{3}$ at $\mathrm{C}-2$ of benzimidazole $), 7.77-7.56(\mathrm{~m}, 4 \mathrm{H}$, aromatic protons $), 8.49(t, 1 \mathrm{H},-\mathrm{N}=\mathrm{CH}) ; \mathrm{EI}-\mathrm{MS}$ : $231\left(\mathrm{M}^{+}+1\right)$.

2.2c $\quad N^{\prime}$-Butylidene-1-carbohydrazido-2-methyl-1Hbenzimidazole (4c): IR (KBr): $1610(-\mathrm{C}=\mathrm{N}), 1590$ $(-\mathrm{N}=\mathrm{CH}), \quad 1677 \quad(-\mathrm{C}=\mathrm{O}$ amido $), 3323 \quad(-\mathrm{C}-\mathrm{NH})$ $\mathrm{cm}^{-1} ;{ }^{1} \mathrm{H}-\mathrm{NMR}$ (DMSO- $\left.d_{6}, \delta \mathrm{ppm}\right): 0.99(t, 3 \mathrm{H}$, $\left.-\mathrm{CHCH}_{2} \mathrm{CH}_{2} \mathrm{CH}_{3}\right), 1.39\left(\mathrm{~m}, 2 \mathrm{H},-\mathrm{CHCH}_{2} \mathrm{CH}_{2} \mathrm{CH}_{3}\right)$, $1.54\left(m, 2 \mathrm{H},-\mathrm{CHCH}_{2} \mathrm{CH}_{2} \mathrm{CH}_{3}\right), 2.46\left(s, 3 \mathrm{H},-\mathrm{CH}_{3}\right.$ at $\mathrm{C}-2$ of benzimidazole $), 3.96\left(\mathrm{~m}, 1 \mathrm{H},-\mathrm{CHCH}_{2}\right.$ $\left.\mathrm{CH}_{2} \mathrm{CH}_{3}\right), 7.82-7.60(m, 4 \mathrm{H}$, aromatic protons); EI-MS: $245\left(\mathrm{M}^{+}+1\right)$. 
2.2d 2-Methyl-N'-(phenylmethylidene)-1-carbohydrazido-1H-benzimidazole (4d): IR (KBr): 1610 $(-\mathrm{C}=\mathrm{N}), 1590(-\mathrm{N}=\mathrm{CH}), 1677(-\mathrm{C}=\mathrm{O}$ amido $), 3323$ $(-\mathrm{C}-\mathrm{NH}) \mathrm{cm}^{-1} ;{ }^{1} \mathrm{H}-\mathrm{NMR}\left(\mathrm{CDCl}_{3}, \delta \mathrm{ppm}\right): 2.39$ $\left(s, 3 \mathrm{H},-\mathrm{CH}_{3}\right.$ at $\mathrm{C}-2$ of benzimidazole), 7.96-7.70 $(m, 9 \mathrm{H}$, aromatic protons) $8.51(s, 1 \mathrm{H},-\mathrm{N}=\mathrm{CH})$; EI-MS: $279\left(\mathbf{M}^{+}+1\right)$.

\section{2e 2-Methyl-N'-[(2-methylphenyl)methylidene $]-$} 1-carbohydrazido-1H-benzimidazole (4e): IR (KBr): $1617(-\mathrm{C}=\mathrm{N}), 1594(-\mathrm{N}=\mathrm{CH}), 1680(-\mathrm{C}=\mathrm{O}$ amido $)$, $3310(-\mathrm{C}-\mathrm{NH}) \mathrm{cm}^{-1} ;{ }^{1} \mathrm{H}-\mathrm{NMR}\left(\mathrm{CDCl}_{3}, \delta \mathrm{ppm}\right): 2 \cdot 29$ $\left(s, 3 \mathrm{H},-\mathrm{CH}_{3}\right.$ at $\mathrm{C}-2$ of benzimidazole $), 2 \cdot 46(s, 3 \mathrm{H}$, $-\mathrm{CH}_{3}$ at benzene ring), 7.87-7.61 $(\mathrm{m}, 8 \mathrm{H}$, aromatic protons $), \quad 8.42(s, \quad 1 \mathrm{H}, \quad-\mathrm{N}=\mathrm{CH}) ; \quad$ EI-MS: 293 $\left(\mathrm{M}^{+}+1\right)$.

2.2f 2-Methyl-N'-[(3-methylphenyl)methylidene $]-1-$ carbohydrazido-1H-benzimidazole (4f): IR (KBr): $1612(-\mathrm{C}=\mathrm{N}), 1580(-\mathrm{N}=\mathrm{CH}), 1689(-\mathrm{C}=\mathrm{O}$ amido $)$, $3313(-\mathrm{C}-\mathrm{NH}) \mathrm{cm}^{-1} ;{ }^{1} \mathrm{H}-\mathrm{NMR}\left(\mathrm{CDCl}_{3}, \delta \mathrm{ppm}\right): 2 \cdot 12$ $\left(s, 3 \mathrm{H},-\mathrm{CH}_{3}\right.$ at benzene ring), $2.32\left(s, 3 \mathrm{H},-\mathrm{CH}_{3}\right.$ at $\mathrm{C}-2$ of benzimidazole), 7.79-7.51 ( $\mathrm{m}, 8 \mathrm{H}$, aromatic protons $), \quad 8.71 \quad(s, \quad 1 \mathrm{H}, \quad-\mathrm{N}=\mathrm{CH}) ; \quad \mathrm{EI}-\mathrm{MS}: 293$ $\left(\mathrm{M}^{+}+1\right)$.

\section{$2.2 \mathrm{~g} \mathrm{~N}$-[(2-Chlorophenyl)methylidene]-1-carbo-} hydrazido-2-methyl-1H-benzimidazole (4g): $\quad \mathrm{IR}(\mathrm{KBr})$ : $1624(-\mathrm{C}=\mathrm{N}), 1587(-\mathrm{N}=\mathrm{CH}), 1682(-\mathrm{C}=\mathrm{O}$ amido $)$, $3315(-\mathrm{C}-\mathrm{NH}) \mathrm{cm}^{-1} ;{ }^{1} \mathrm{H}-\mathrm{NMR}\left(\mathrm{CDCl}_{3}, \delta \mathrm{ppm}\right)$ : $2.40\left(s, 3 \mathrm{H},-\mathrm{CH}_{3}\right.$ at $\mathrm{C}-2$ of benzimidazole $), 7.84$ $7.50(m, 8 \mathrm{H}$, aromatic protons $), 8.60(s, 1 \mathrm{H}$, $-\mathrm{N}=\mathrm{CH})$; EI-MS: $313\left(\mathrm{M}^{+}+1\right)$.

\section{2h $\quad N^{\prime}-[(4-C h l o r o p h e n y l) m e t h y l i d e n e]-1$-carbo-} hydrazido-2-methyl-1H-benzimidazole (4h): $\quad \mathrm{IR}(\mathrm{KBr})$ : $1623(-\mathrm{C}=\mathrm{N}), 1595(-\mathrm{N}=\mathrm{CH}), 1698(-\mathrm{C}=\mathrm{O}$ amido $)$, $3324(-\mathrm{C}-\mathrm{NH}) \mathrm{cm}^{-1} ;{ }^{1} \mathrm{H}-\mathrm{NMR}\left(\mathrm{CDCl}_{3}, \delta \mathrm{ppm}\right): 2 \cdot 38$ $\left(s, 3 \mathrm{H},-\mathrm{CH}_{3}\right.$ at $\mathrm{C}-2$ of benzimidazole), $7.98-7.72$ ( $m, 8 \mathrm{H}$, aromatic protons), $8.45(s, 1 \mathrm{H},-\mathrm{N}=\mathrm{CH})$; EI-MS: $313\left(\mathrm{M}^{+}+1\right)$.

$2.2 \mathrm{i} \quad N^{\prime}-[(2-H y d r o x y p h e n y l)$ methylidene]-1-carbohydrazido-2-methyl-1H-benzimidazole (4i): IR (KBr): $1627(-\mathrm{C}=\mathrm{N}), 1574(-\mathrm{N}=\mathrm{CH}), 1679(-\mathrm{C}=\mathrm{O}$ amido $)$, $3328 \quad(-\mathrm{C}-\mathrm{NH}) \quad \mathrm{cm}^{-1}$; ${ }^{1} \mathrm{H}-\mathrm{NMR} \quad$ (DMSO- $d_{6}$, $\delta \mathrm{ppm}): 2.42\left(s, 3 \mathrm{H},-\mathrm{CH}_{3}\right.$ at $\mathrm{C}-2$ of benzimidazole $)$, $5.82(s, 1 \mathrm{H}, \mathrm{Ar}-\mathrm{OH}), 7.73-7.59(\mathrm{~m}, 8 \mathrm{H}$, aromatic protons $), \quad 8.52(s, \quad 1 \mathrm{H}, \quad-\mathrm{N}=\mathrm{CH}) ; \quad$ EI-MS: 295 $\left(\mathrm{M}^{+}+1\right)$.
$2.2 \mathrm{j} \quad N^{\prime}-[(3-H y d r o x y p h e n y l) m e t h y l i d e n e]-1$-carbohydrazido-2-methyl-1H-benzimidazole $(\mathbf{4 j})$ : $\mathrm{IR}(\mathrm{KBr})$ : $1628(-\mathrm{C}=\mathrm{N}), 1580(-\mathrm{N}=\mathrm{CH}), 1677(-\mathrm{C}=\mathrm{O}$ amido $)$, $3323(-\mathrm{C}-\mathrm{NH}) \mathrm{cm}^{-1} ;{ }^{1} \mathrm{H}-\mathrm{NMR}$ (DMSO- $\left.d_{6}, \delta \mathrm{ppm}\right)$ : $2.48\left(s, 3 \mathrm{H},-\mathrm{CH}_{3}\right.$ at $\mathrm{C}-2$ of benzimidazole), 5.55 $(s, 1 \mathrm{H}, \mathrm{Ar}-\mathrm{OH}), 7 \cdot 80-7.62(\mathrm{~m}, 8 \mathrm{H}$, aromatic protons), $8.49(s, 1 \mathrm{H},-\mathrm{N}=\mathrm{CH})$; EI-MS: $295\left(\mathrm{M}^{+}+1\right)$.

$2.2 \mathrm{k} \quad N^{\prime}-[(4-H y d r o x y p h e n y l) m e t h y l i d e n e]-1-c a r b o-$ hydrazido-2-methyl-1H-benzimidazole (4k): IR $(\mathrm{KBr}): 1625(-\mathrm{C}=\mathrm{N}), 1576(-\mathrm{N}=\mathrm{CH}), 1682(-\mathrm{C}=\mathrm{O}$ amido), 3327 (-C-NH) cm ${ }^{-1} ;{ }^{1} \mathrm{H}-\mathrm{NMR}$ (DMSO- $d_{6}, \delta$ ppm): $2.40\left(s, 3 \mathrm{H},-\mathrm{CH}_{3}\right.$ at $\mathrm{C}-2$ of benzimidazole $)$, $5.75(s, 1 \mathrm{H}, \mathrm{Ar}-\mathrm{OH}), 7.83-7.62(\mathrm{~m}, 8 \mathrm{H}$, aromatic protons $), \quad 8.42(s, \quad 1 \mathrm{H}, \quad-\mathrm{N}=\mathrm{CH}) ; \quad \mathrm{EI}-\mathrm{MS}: 295$ $\left(\mathrm{M}^{+}+1\right)$.

$2.21 \quad N^{\prime}-[(2-M e t h o x y p h e n y l)$ methylidene]-1-carbohydrazido-2-methyl-1H-benzimidazole (4I): $\mathrm{IR}(\mathrm{KBr})$ : $1618(-\mathrm{C}=\mathrm{N}), 1592(-\mathrm{N}=\mathrm{CH}), 1673(-\mathrm{C}=\mathrm{O}$ amido), $3320(-\mathrm{C}-\mathrm{NH}) \mathrm{cm}^{-1},{ }^{1} \mathrm{H}-\mathrm{NMR}\left(\mathrm{CDCl}_{3}, \delta \mathrm{ppm}\right): 2 \cdot 39$ $\left(s, 3 \mathrm{H},-\mathrm{CH}_{3}\right.$ at $\mathrm{C}-2$ of benzimidazole $), 3.75(s, 3 \mathrm{H}$, $\left.-\mathrm{OCH}_{3}\right), 7.97-7.70(\mathrm{~m}, 8 \mathrm{H}$, aromatic protons), 8.60 $(s, 1 \mathrm{H},-\mathrm{N}=\mathrm{CH})$; EI-MS: $309\left(\mathrm{M}^{+}+1\right)$.

$2.2 \mathrm{~m} \quad N^{\prime}-[(4-M e t h o x y p h e n y l)$ methylidene]-1-carbohydrazido-2-methyl-1H-benzimidazole $\quad(\mathbf{4 m})$ : IR $(\mathrm{KBr}): 1617(-\mathrm{C}=\mathrm{N}), 1595(-\mathrm{N}=\mathrm{CH}), 1680 \quad(-\mathrm{C}=\mathrm{O}$ amido), $3322(-\mathrm{C}-\mathrm{NH}) \mathrm{cm}^{-1} ;{ }^{1} \mathrm{H}-\mathrm{NMR}\left(\mathrm{CDCl}_{3}, \delta\right.$ ppm): $2.35\left(s, 3 \mathrm{H},-\mathrm{CH}_{3}\right.$ at $\mathrm{C}-2$ of benzimidazole $)$, $3.64\left(s, 3 \mathrm{H},-\mathrm{OCH}_{3}\right), 7.90-7.65(\mathrm{~m}, 8 \mathrm{H}$, aromatic protons $), \quad 8.57 \quad(s, \quad 1 \mathrm{H}, \quad-\mathrm{N}=\mathrm{CH}) ; \quad$ EI-MS: 309 $\left(\mathrm{M}^{+}+1\right)$.

$2.3 \mathrm{~N}$-[3-Chloro-2-(substituted) alkyl/aryl-4oxoazetidin-1-yl]-1-carboxamido-2-methyl-1Hbenzimidazoles (5a-5m)

General procedure: To a stirred solution of compound (4a-4m) (20 mmol) and triethylamine $(10 \mathrm{mmol})$ in dry dioxane $(50 \mathrm{ml})$ was added chloroacetyl chloride $(10 \mathrm{mmol})$ dropwise at room temperature. The reaction mixture was stirred for $1 \mathrm{~h}$ and then refluxed for 5-10 h. The solid obtained after removal of dioxane was crystallized from aq. ethanol to give the title compounds.

2.3a N-(3-Chloro-2-methyl-4-oxoazetidin-1-yl)-1carboxamido-2-methyl-1H-benzimidazole (5a): IR $(\mathrm{KBr}): 1618 \quad(-\mathrm{C}=\mathrm{N}), 1670 \quad(-\mathrm{C}=\mathrm{O}$ amido $), 1726$ $(>\mathrm{C}=\mathrm{O}$ monocyclic $\beta$-lactam), $3318(-\mathrm{C}-\mathrm{NH}), 772$ 
$(-\mathrm{C}-\mathrm{Cl}) \mathrm{cm}^{-1} ;{ }^{1} \mathrm{H}-\mathrm{NMR}$ (DMSO- $\left.d_{6}, \delta \mathrm{ppm}\right): 1.30(d$, $3 \mathrm{H},-\mathrm{CH}_{3}$ of side chain at $\beta$-lactam $), 2.45(s, 3 \mathrm{H}$, $-\mathrm{CH}_{3}$ at $\mathrm{C}-2$ of benzimidazole $), 4.11(p, 1 \mathrm{H}$, $-\mathrm{CHCH}_{3}, J=7.01,7.01,7.03$ and $\left.7.05 \mathrm{~Hz}\right), 5.44$ $(d, 1 \mathrm{H},-\mathrm{CHCl}, J=6.96 \mathrm{~Hz}), 7.82-7.71(\mathrm{~m}, 4 \mathrm{H}$, aromatic protons); EI-MS: $293\left(\mathrm{M}^{+}+1\right)$.

2.3b N-(3-Chloro-2-ethyl-4-oxoazetidin-1-yl)-1-carboxamido-2-methyl-1H-benzimidazole (5b): $\mathrm{IR}(\mathrm{KBr})$ : $1620(-\mathrm{C}=\mathrm{N}), 1676(-\mathrm{C}=\mathrm{O}$ amido $), 1720(>\mathrm{C}=\mathrm{O}$ monocyclic $\beta$-lactam), $3319(-\mathrm{C}-\mathrm{NH}), 763(-\mathrm{C}-\mathrm{Cl})$ $\mathrm{cm}^{-1} ;{ }^{1} \mathrm{H}-\mathrm{NMR}$ (DMSO- $\left.d_{6}, \delta \mathrm{ppm}\right): \delta 1.00(t, 3 \mathrm{H}$, $\left.-\mathrm{CH}_{2} \mathrm{CH}_{3}\right), 1.45\left(p, 2 \mathrm{H},-{ }_{-1} \mathrm{CH}_{2} \mathrm{CH}_{3}\right), 2.43(s, 3 \mathrm{H}$, $-\mathrm{CH}_{3}$ at $\mathrm{C}-2$ of benzimidazole $), 3.98(q, 1 \mathrm{H},-\mathrm{CH}$ of $\beta$-lactam, $J=7 \cdot 16,7 \cdot 16$ and $7 \cdot 20 \mathrm{~Hz}), 5.41(d, 1 \mathrm{H}$, $-\mathrm{CHCl}, J=6.96 \mathrm{~Hz}), 7.80-7.62(m, 4 \mathrm{H}$, aromatic protons); EI-MS: $307\left(\mathrm{M}^{+}+1\right)$.

2.3c N-(3-Chloro-2-oxo-4-propylazetidin-1-yl)-1carboxamido-2-methyl-1H-benzimidazole (5c): IR $(\mathrm{KBr}): 1616(-\mathrm{C}=\mathrm{N}), 1673 \quad(-\mathrm{C}=\mathrm{O}$ amido $), 1723$ $(>\mathrm{C}=\mathrm{O}$ monocyclic $\beta$-lactam $), 3317(-\mathrm{C}-\mathrm{NH}), 780$ $(-\mathrm{C}-\mathrm{Cl}) \mathrm{cm}^{-1} ;{ }^{1} \mathrm{H}-\mathrm{NMR}$ (DMSO- $\left.d_{6}, \delta \mathrm{ppm}\right): 0.98(t$, $\left.3 \mathrm{H},-\mathrm{CH}_{2} \mathrm{CH}_{2} \mathrm{CH}_{3}\right), 1.38\left(m, 2 \mathrm{H},-\mathrm{CH}_{2} \mathrm{CH}_{2} \mathrm{CH}_{3}\right)$, $1.56\left(q, 2 \mathrm{H}, \mathrm{CH}_{2} \mathrm{CH}_{2} \mathrm{CH}_{3}\right), 2.49\left(s, 3 \mathrm{H},-\mathrm{CH}_{3}\right.$ at $\mathrm{C}-2$ of benzimidazole $), 3.90(q, 1 \mathrm{H},-\mathrm{CH}$ of $\beta$-lactam, $J=7.12,7.12$ and $7.15 \mathrm{~Hz}), 5.48(d, 1 \mathrm{H},-\mathrm{CHCl}$, $J=7.00 \mathrm{~Hz}), 7.76-7.61$ ( $m, 4 \mathrm{H}$, aromatic protons); EI-MS: $321\left(\mathrm{M}^{+}+1\right)$.

2.3d N-(3-Chloro-2-oxo-4-phenylazetidin-1-yl)-1carboxamido-2-methyl-1H-benzimidazole (5d): IR $(\mathrm{KBr}): 1625(-\mathrm{C}=\mathrm{N}), 1687 \quad(-\mathrm{C}=\mathrm{O}$ amido $), 1730$ $(>\mathrm{C}=\mathrm{O}$ monocyclic $\beta$-lactam), $3324(-\mathrm{C}-\mathrm{NH}), 772$ $(-\mathrm{C}-\mathrm{Cl}) \mathrm{cm}^{-1} ;{ }^{1} \mathrm{H}-\mathrm{NMR} \quad\left(\mathrm{CDCl}_{3}, \delta \mathrm{ppm}\right): 2.37$ $\left(s, 3 \mathrm{H},-\mathrm{CH}_{3}\right.$ at $\mathrm{C}-2$ of benzimidazole $), 5.02(d, 1 \mathrm{H}$, $-\mathrm{CH}$ of $\beta$-lactam, $J=8.6 \mathrm{~Hz}), 5.25(d, 1 \mathrm{H},-\mathrm{CHCl}$, $J=7.9 \mathrm{~Hz}), 7.94-7.65(m, 9 \mathrm{H}$, aromatic protons $)$; EI-MS: $355\left(\mathrm{M}^{+}+1\right)$.

2.3e N-[3-chloro-2-(2-methylphenyl)-4-oxoazetidin1-yl]-1-carboxamido-2-methyl-1H-benzimidazole

(5e): IR (KBr): $1630(-\mathrm{C}=\mathrm{N}), 1680(-\mathrm{C}=\mathrm{O}$ amido), $1716(>\mathrm{C}=\mathrm{O}$ monocyclic $\beta$-lactam $), 3340(-\mathrm{C}-\mathrm{NH})$, $760(-\mathrm{C}-\mathrm{Cl}) \mathrm{cm}^{-1} ;{ }^{1} \mathrm{H}-\mathrm{NMR}\left(\mathrm{CDCl}_{3}, \delta \mathrm{ppm}\right): \delta 2 \cdot 34$ $\left(s, 3 \mathrm{H},-\mathrm{CH}_{3}\right.$ at benzene), $2.40\left(s, 3 \mathrm{H},-\mathrm{CH}_{3}\right.$ at $\mathrm{C}-2$ of benzimidazole $), 5.05(d, 1 \mathrm{H},-\mathrm{CH}$ of $\beta$-lactam, $J=8.7 \mathrm{~Hz}), \quad 5.45 \quad(d, \quad 1 \mathrm{H}, \quad-\mathrm{CHCl}, \quad J=7.9 \mathrm{~Hz})$, 7.90-7.74 ( $m, 8 \mathrm{H}$, aromatic protons); EI-MS: 369 $\left(\mathrm{M}^{+}+1\right)$.
$2.3 \mathrm{f} \quad \mathrm{N}$-[3-Chloro-2-(3-methylphenyl)-4-oxoazetidin1-yl]-1-carboxamido-2-methyl-1H-benzimidazole

(5f): $\quad \mathrm{IR}(\mathrm{KBr}): 1611(-\mathrm{C}=\mathrm{N}), 1682(-\mathrm{C}=\mathrm{O}$ amido), $1727(>\mathrm{C}=\mathrm{O}$ monocyclic $\beta$-lactam), $3318(-\mathrm{C}-\mathrm{NH})$, $758(-\mathrm{C}-\mathrm{Cl}) \mathrm{cm}^{-1} ;{ }^{1} \mathrm{H}-\mathrm{NMR}\left(\mathrm{CDCl}_{3}, \delta \mathrm{ppm}\right): 2 \cdot 38$ $\left(s, 3 \mathrm{H},-\mathrm{CH}_{3}\right.$ at benzene), $2.44\left(s, 3 \mathrm{H},-\mathrm{CH}_{3}\right.$ at $\mathrm{C}-2$ of benzimidazole $), 4.99(d, 1 \mathrm{H},-\mathrm{CH}$ of $\beta$-lactam, $J=8.4 \mathrm{~Hz}), \quad 5.42 \quad(d, \quad 1 \mathrm{H}, \quad-\mathrm{CHCl}, \quad J=7.2 \mathrm{~Hz})$, 7.87-7.61 ( $m, 8 \mathrm{H}$, aromatic protons); EI-MS: 369 $\left(\mathrm{M}^{+}+1\right)$.

$2.3 \mathrm{~g} \quad \mathrm{~N}$-[3-Chloro-2-(2-chlorophenyl)-4-oxoazetidin1-yl]-1-carboxamido-2-methyl-1H-benzimidazole $\quad \mathbf{( 5 g})$ : IR (KBr): $1634(-\mathrm{C}=\mathrm{N}), 1690(-\mathrm{C}=\mathrm{O}$ amido $), 1733$ ( $>\mathrm{C}=\mathrm{O}$ monocyclic $\beta$-lactam), $3327(-\mathrm{C}-\mathrm{NH}), 765$ $(-\mathrm{C}-\mathrm{Cl}) \mathrm{cm}^{-1} ;{ }^{1} \mathrm{H}-\mathrm{NMR}$ (DMSO- $d_{6}, \delta \mathrm{ppm}: 2.42(s$, $3 \mathrm{H},-\mathrm{CH}_{3}$ at $\mathrm{C}-2$ of benzimidazole $), 5.02(d, 1 \mathrm{H}$, -CH of $\beta$-lactam, $J=8.7 \mathrm{~Hz}), 5.44(d, 1 \mathrm{H},-\mathrm{CHCl}$, $J=7.7 \mathrm{~Hz}), 7.69-7.41(m, 8 \mathrm{H}$, aromatic protons); EI - MS: $389\left(M^{+}+1\right)$.

$2.3 \mathrm{~h} \quad \mathrm{~N}$-[3-Chloro-2-(4-chlorophenyl)-4-oxoazetidin-

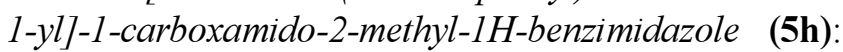
IR (KBr): $1617(-\mathrm{C}=\mathrm{N}), 1679(-\mathrm{C}=\mathrm{O}$ amido), 1729 $(>\mathrm{C}=\mathrm{O}$ monocyclic $\beta$-lactam), $3312(-\mathrm{C}-\mathrm{NH}), 769$ $(-\mathrm{C}-\mathrm{Cl}) \mathrm{cm}^{-1} ;{ }^{1} \mathrm{H}-\mathrm{NMR}\left(\mathrm{CDCl}_{3}, \delta \mathrm{ppm}\right): \delta 2.39(s$, $3 \mathrm{H},-\mathrm{CH}_{3}$ at $\mathrm{C}-2$ of benzimidazole $), 5.02(d, 1 \mathrm{H}$, $-\mathrm{CH}$ of $\beta$-lactam, $J=8.6 \mathrm{~Hz}), 5.40(d, 1 \mathrm{H},-\mathrm{CHCl}$, $J=7.5 \mathrm{~Hz}), 7.79-7.54(m, 8 \mathrm{H}$, aromatic protons $)$; EI-MS: $389\left(\mathrm{M}^{+}+1\right)$.

$2.3 \mathrm{i} \quad \mathrm{N}$-[3-Chloro-2-(2-hydroxyphenyl)-4-oxoazetidin1-yl]-1-carboxamido-2-methyl-1H-benzimidazole (5i): IR $(\mathrm{KBr}): 1613 \quad(-\mathrm{C}=\mathrm{N}), 1677 \quad(-\mathrm{C}=\mathrm{O}$ amido), $1728(>\mathrm{C}=\mathrm{O}$ monocyclic $\beta$-lactam $), 3313 \quad(-\mathrm{C}-$ $\mathrm{NH}), 770(-\mathrm{C}-\mathrm{Cl}) \mathrm{cm}^{-1} ;{ }^{1} \mathrm{H}-\mathrm{NMR}\left(\mathrm{CDCl}_{3}, \delta \mathrm{ppm}\right)$ : $2.46\left(s, 3 \mathrm{H},-\mathrm{CH}_{3}\right.$ at $\mathrm{C}-2$ of benzimidazole $), 4.97$ $(d, 1 \mathrm{H},-\mathrm{CH}$ of $\beta$-lactam, $J=8.0 \mathrm{~Hz}), 5.42(d$, $1 \mathrm{H},-\mathrm{CHCl}, J=6.8 \mathrm{~Hz}), 5.58(s, 1 \mathrm{H}, \mathrm{Ar}-\mathrm{OH}), 7.84-$ $7.61(m, 8 \mathrm{H}$, aromatic protons); EI-MS: 371 $\left(\mathrm{M}^{+}+1\right)$.

$2.3 \mathrm{j} \quad \mathrm{N}$-[3-Chloro-2-(3-hydroxyphenyl)-4-oxoazetidin1-yl]-1-carboxamido-2-methyl-1H-benzimidazole (5j): IR (KBr): $1618(-\mathrm{C}=\mathrm{N}), 1668(-\mathrm{C}=\mathrm{O}$ amido $), 1729$ $(>\mathrm{C}=\mathrm{O}$ monocyclic $\beta$-lactam), $3318(-\mathrm{C}-\mathrm{NH}), 768$ $(-\mathrm{C}-\mathrm{Cl}) \mathrm{cm}^{-1} ;{ }^{1} \mathrm{H}-\mathrm{NMR}$ (DMSO- $\left.d_{6}, \delta \mathrm{ppm}\right): 2.44$ $\left(s, 3 \mathrm{H},-\mathrm{CH}_{3}\right.$ at $\mathrm{C}-2$ of benzimidazole), $4.88(d, 1 \mathrm{H}$, $-\mathrm{CH}$ of $\beta$-lactam, $J=7.9 \mathrm{~Hz}), 5.39(d, 1 \mathrm{H},-\mathrm{CHCl}$, $J=7.1 \mathrm{~Hz}), 5.73(s, 1 \mathrm{H}, \mathrm{Ar}-\mathrm{OH}), 7.87-7.62(m, 8 \mathrm{H}$, aromatic protons); EI-MS: $371\left(\mathbf{M}^{+}+1\right)$. 
$2.3 \mathrm{k} \quad \mathrm{N}$-[3-Chloro-2-(4-hydroxyphenyl)-4-oxoazetidin1-yl]-1-carboxamido-2-methyl-1H-benzimidazole (5k): IR (KBr): $1617(-\mathrm{C}=\mathrm{N}), 1674(-\mathrm{C}=\mathrm{O}$ amido), 1730 $(>\mathrm{C}=\mathrm{O}$ monocyclic $\beta$-lactam), $3323(-\mathrm{C}-\mathrm{NH}), 761$ $(-\mathrm{C}-\mathrm{Cl}) \mathrm{cm}^{-1} ;{ }^{1} \mathrm{H}-\mathrm{NMR}$ (DMSO- $\left.d_{6}, \delta \mathrm{ppm}\right): 2.49$ $\left(s, 3 \mathrm{H},-\mathrm{CH}_{3}\right.$ at $\mathrm{C}-2$ of benzimidazole $), 4.96(d, 1 \mathrm{H}$, $-\mathrm{CH}$ of $\beta$-lactam, $J=8.2 \mathrm{~Hz}), 5.45(d, 1 \mathrm{H},-\mathrm{CHCl}$, $J=7 \cdot 1 \mathrm{~Hz}), 5.65(s, 1 \mathrm{H}, \mathrm{Ar}-\mathrm{OH}), 7 \cdot 82-7.61(\mathrm{~m}, 8 \mathrm{H}$, aromatic protons); EI-MS: $371\left(\mathrm{M}^{+}+1\right)$.

$2.31 \quad N$-[3-Chloro-2-(2-methoxyphenyl)-4-oxoazetidin1-yl]-1-carboxamido-2-methyl-1H-benzimidazole (51): IR (KBr): $1624(-\mathrm{C}=\mathrm{N}), 1687(-\mathrm{C}=\mathrm{O}$ amido), 1734 $(>\mathrm{C}=\mathrm{O}$ monocyclic $\beta$-lactam), $3299(-\mathrm{C}-\mathrm{NH}), 759$ $(-\mathrm{C}-\mathrm{Cl}) \mathrm{cm}^{-1} ;{ }^{1} \mathrm{H}-\mathrm{NMR} \quad\left(\mathrm{CDCl}_{3}, \delta \mathrm{ppm}\right): 2.41$ $\left(s, 3 \mathrm{H},-\mathrm{CH}_{3}\right.$ at $\mathrm{C}-2$ of benzimidazole), $3.73(s, 3 \mathrm{H}$, $\left.-\mathrm{OCH}_{3}\right), 4.96(d, 1 \mathrm{H},-\mathrm{CH}$ of $\beta$-lactam, $J=8.0 \mathrm{~Hz})$, $5.42(d, 1 \mathrm{H},-\mathrm{CHCl}, J=6.95 \mathrm{~Hz}), 7.71-7.52(m, 8 \mathrm{H}$, aromatic protons); EI-MS: $385\left(\mathbf{M}^{+}+1\right)$.

$2.3 \mathrm{~m} \quad \mathrm{~N}$-[3-Chloro-2-(4-methoxyphenyl)-4-oxoazetidin-1-yl]-1-carboxamido-2-methyl-1H-benzimidazole $(\mathbf{5 m})$ : IR $(\mathrm{KBr}): 1629(-\mathrm{C}=\mathrm{N}), 1693 \quad(-\mathrm{C}=\mathrm{O}$ amido), 1731 ( $>\mathrm{C}=\mathrm{O}$ monocyclic $\beta$-lactam $), 3305$ $(-\mathrm{C}-\mathrm{NH}), 763(-\mathrm{C}-\mathrm{Cl}) . \mathrm{cm}^{-1} ;{ }^{1} \mathrm{H}-\mathrm{NMR}\left(\mathrm{CDCl}_{3}\right.$, $\delta \mathrm{ppm}): 2.45\left(s, 3 \mathrm{H},-\mathrm{CH}_{3}\right.$ at $\mathrm{C}-2$ of benzimidazole), $3.78\left(s, 3 \mathrm{H},-\mathrm{OCH}_{3}\right), 4.92(d, 1 \mathrm{H},-\mathrm{CH}$ of $\beta$-lactam, $J=8.0 \mathrm{~Hz}), 5.46(d, 1 \mathrm{H},-\mathrm{CHCl}, J=6.8 \mathrm{~Hz}), 7.75-$ $7.58(\mathrm{~m}, 8 \mathrm{H}$, aromatic protons $) ;$ EI-MS: 385 $\left(\mathrm{M}^{+}+1\right)$.

\subsection{Antimicrobial activity test}

The compounds $(\mathbf{5} \mathbf{a}-\mathbf{5 m})$ were tested for their in vitro growth inhibitory activity against different microbes. The bacterial strains used were Staphylococcus aureus ATCC 29213, Streptococcus mutans MTCC 890 and Bacillus subtilis MTCC 741 (all Gram positive) and Ecsherichia coli ATCC 25922, Salmonella typhi MTCC 733 and Pseudomonas aeruginosa MTCC 741 (all Gram negative). For testing the antifungal activity of the synthesized compounds the fungal strains Candida albicans MTCC 1637, Aspergillus flavus AIIMS and Aspergillus niger AIIMS were used.

The inhibition zones of synthesized compounds were determined using disc diffusion method. ${ }^{31}$ In this method, paper disks $(6 \mathrm{~mm})$ containing specific amounts of an antimicrobial agent (300 $\mu \mathrm{g}$ for the synthesized compounds) were placed on the surface of an agar plate inoculated with a standardized sus- pension of the microorganisms tested. The plates were incubated at $35^{\circ} \mathrm{C}$ for 24 and $48 \mathrm{~h}$, respectively for bacteria and fungi. Ampicillin $(10 \mu \mathrm{g})$ for Gram positive bacteria, Nalidixic acid $(30 \mu \mathrm{g})$ for Gram negative bacteria and Amphotericin B (30 $\mu \mathrm{g})$ for fungi, were used as standard drugs. Paper disks with only DMSO were utilized as negative control. All experiments were carried out three times. The inhibition zones produced by the various synthesized compounds on the microbial growth were measured (diameter in $\mathrm{mm}$ ). Physical and analytical data of the synthesized compounds are reported in table 1. The data on antimicrobial activity of compounds $(\mathbf{5 a}-\mathbf{5 m})$ are shown in tables 2 and 3 .

\section{Results and discussion}

\subsection{Chemistry}

The reaction sequenced for different title compounds is outlined in scheme 1. 2-Methyl- $1 \mathrm{H}$-benzimidazole 1 and ethyl 2-methyl- $1 H$-benzimidazole1 -carboxylate 2 were prepared according to the literature procedure. ${ }^{32}$ Compound $\mathbf{2}$ on treatment with hydrazine hydrate in ethanol yielded 2-methyl- $1 \mathrm{H}$ benzimidazole-1-carbohydrazide $\mathbf{3}$. Compound $\mathbf{3}$ on condensation with various aldehydes furnished 2-methyl- $N^{\prime}$-[(substituted) alkyl/aryl methylidene]1 -carbohydrazido- $1 H$-benzimidazoles (4a-4m). The four-membered $\beta$-lactam ring was introduced in compounds $(\mathbf{4 a}-\mathbf{4 m})$ at the azomethine group by the cycloaddition of chloroacetyl chloride in the presence of triethylamine, according to literature, ${ }^{7}$ to yield $N$-[3-chloro-2-(substituted) alkyl/aryl-4oxoazetidin-1-yl]-1-carboxamido-2-methyl-1H-benzimidazoles $(\mathbf{5 a}-\mathbf{m})$. The purity of the compounds was monitored by TLC. The structural evaluation of the newly synthesized derivatives and of the intermediates synthesized in this study were performed using IR, ${ }^{1} \mathrm{H}-\mathrm{NMR}$ and mass spectroscopic techniques which are in agreement with the proposed structures.

\subsection{Antimicrobial evaluation}

The in vitro antimicrobial activity was performed using the disc diffusion method with different strains of bacteria and fungi. Ampicillin and nalidixic acid were used as positive control for bacteria and amphotericin B was used against fungi. 
Table 1. Physical and analytical data of the compounds $2,3,(\mathbf{4 a}-\mathbf{4 m})$ and (5a-5m).

\begin{tabular}{|c|c|c|c|c|c|c|c|}
\hline \multirow[b]{2}{*}{ Compds } & \multirow[b]{2}{*}{$\mathrm{R}$} & \multirow[b]{2}{*}{ Yield (\%) } & \multirow[b]{2}{*}{ m.p. $\left({ }^{\circ} \mathrm{C}\right)$} & \multirow{2}{*}{$\begin{array}{l}\text { Mol·formula } \\
\text { (formula wt.) }\end{array}$} & \multicolumn{3}{|c|}{ Analysis $\%$ found (calculated) } \\
\hline & & & & & $\mathrm{C}$ & $\mathrm{H}$ & $\mathrm{N}$ \\
\hline \multirow[t]{2}{*}{2} & \multirow[t]{2}{*}{-} & \multirow[t]{2}{*}{79} & \multirow[t]{2}{*}{$180-82$} & \multirow{2}{*}{$\begin{array}{l}\mathrm{C}_{11} \mathrm{H}_{12} \mathrm{~N}_{2} \mathrm{O}_{2} \\
(204 \cdot 23)\end{array}$} & $64 \cdot 67$ & $5 \cdot 90$ & $13 \cdot 70$ \\
\hline & & & & & $(64 \cdot 69$ & $5 \cdot 92$ & $13 \cdot 72)$ \\
\hline \multirow[t]{2}{*}{3} & \multirow[t]{2}{*}{-} & \multirow[t]{2}{*}{78} & \multirow[t]{2}{*}{$173-75$} & $\mathrm{C}_{9} \mathrm{H}_{10} \mathrm{~N}_{4} \mathrm{O}$ & $56 \cdot 80$ & $5 \cdot 27$ & $29 \cdot 42$ \\
\hline & & & & $(190 \cdot 20)$ & $(56 \cdot 83$ & $5 \cdot 30$ & $29 \cdot 46)$ \\
\hline \multirow[t]{2}{*}{$4 \mathbf{a}$} & $-\mathrm{CH}_{3}$ & 82 & $185-87$ & $\mathrm{C}_{11} \mathrm{H}_{12} \mathrm{~N}_{4} \mathrm{O}$ & 61.07 & $5 \cdot 57$ & $25 \cdot 88$ \\
\hline & & & & $(216 \cdot 24)$ & $(61 \cdot 10$ & $5 \cdot 59$ & 25.91) \\
\hline $4 b$ & $-\mathrm{CH}_{2} \mathrm{CH}_{3}$ & 80 & $184-86$ & $\mathrm{C}_{12} \mathrm{H}_{14} \mathrm{~N}_{4} \mathrm{O}$ & $62 \cdot 56$ & $6 \cdot 11$ & $24 \cdot 30$ \\
\hline & & & & $(230 \cdot 27)$ & $(62 \cdot 59$ & $6 \cdot 13$ & $24 \cdot 33)$ \\
\hline 4c & $-\mathrm{CH}_{2} \mathrm{CH}_{2} \mathrm{CH}_{3}$ & 76 & $190-92$ & $\mathrm{C}_{13} \mathrm{H}_{16} \mathrm{~N}_{4} \mathrm{O}$ & $63 \cdot 88$ & $6 \cdot 57$ & $22 \cdot 90$ \\
\hline & & & & $(244 \cdot 29)$ & $(63.91$ & $6 \cdot 60$ & $22 \cdot 93)$ \\
\hline $4 d$ & $-\mathrm{C}_{6} \mathrm{H}_{5}$ & 80 & $180-82$ & $\mathrm{C}_{16} \mathrm{H}_{14} \mathrm{~N}_{4} \mathrm{O}$ & $69 \cdot 03$ & $5 \cdot 04$ & $20 \cdot 11$ \\
\hline & & & & $(278 \cdot 31)$ & $(69.05$ & 5.07 & $20 \cdot 13)$ \\
\hline $4 e$ & $2-\mathrm{CH}_{3} \mathrm{C}_{6} \mathrm{H}_{4}$ & 83 & $183-85$ & $\mathrm{C}_{17} \mathrm{H}_{14} \mathrm{~N}_{4} \mathrm{O}$ & $69 \cdot 81$ & $5 \cdot 50$ & $19 \cdot 15$ \\
\hline & & & & $(292 \cdot 34)$ & $(69 \cdot 85$ & $5 \cdot 52$ & $19 \cdot 17)$ \\
\hline $4 f$ & $3-\mathrm{CH}_{3} \mathrm{C}_{6} \mathrm{H}_{4}$ & 75 & $175-77$ & $\mathrm{C}_{14} \mathrm{H}_{16} \mathrm{ClN}_{4} \mathrm{O}$ & $69 \cdot 82$ & $5 \cdot 48$ & $19 \cdot 15$ \\
\hline & & & & $(292 \cdot 34)$ & $(69 \cdot 85$ & $5 \cdot 52$ & $19 \cdot 17)$ \\
\hline $4 g$ & $2-\mathrm{ClC}_{6} \mathrm{H}_{4}$ & 71 & $169-71$ & $\mathrm{C}_{16} \mathrm{H}_{13} \mathrm{ClN}_{4} \mathrm{O}$ & 61.41 & $4 \cdot 17$ & $17 \cdot 89$ \\
\hline & & & & $(312 \cdot 75)$ & $(61.44$ & $4 \cdot 19$ & 17.91) \\
\hline $4 h$ & $4-\mathrm{ClC}_{6} \mathrm{H}_{4}$ & 69 & $168-70$ & $\mathrm{C}_{16} \mathrm{H}_{13} \mathrm{ClN}_{4} \mathrm{O}$ & 61.43 & $4 \cdot 17$ & $17 \cdot 89$ \\
\hline & & & & $(312.75)$ & $(61.44$ & $4 \cdot 19$ & $17 \cdot 91)$ \\
\hline $4 i$ & $2-\mathrm{OHC}_{6} \mathrm{H}_{4}$ & 85 & $181-83$ & $\mathrm{C}_{16} \mathrm{H}_{14} \mathrm{~N}_{4} \mathrm{O}_{2}$ & $65 \cdot 28$ & $4 \cdot 75$ & $19 \cdot 01$ \\
\hline & & & & $(294 \cdot 31)$ & $(65 \cdot 30$ & $4 \cdot 79$ & $19 \cdot 04)$ \\
\hline $4 j$ & $3-\mathrm{OHC}_{6} \mathrm{H}_{4}$ & 82 & $175-77$ & $\mathrm{C}_{16} \mathrm{H}_{14} \mathrm{~N}_{4} \mathrm{O}_{2}$ & $65 \cdot 27$ & $4 \cdot 74$ & $19 \cdot 02$ \\
\hline & & & & $(294 \cdot 31)$ & $(65 \cdot 30$ & $4 \cdot 79$ & $19 \cdot 04)$ \\
\hline $4 \mathbf{k}$ & $4-\mathrm{OHC}_{6} \mathrm{H}_{4}$ & 81 & $166-68$ & $\mathrm{C}_{16} \mathrm{H}_{14} \mathrm{~N}_{4} \mathrm{O}_{2}$ & $65 \cdot 27$ & $4 \cdot 74$ & $19 \cdot 01$ \\
\hline & & & & $(294 \cdot 31)$ & $(65 \cdot 30$ & $4 \cdot 79$ & $19 \cdot 04)$ \\
\hline 41 & $2-\mathrm{OCH}_{3} \mathrm{C}_{6} \mathrm{H}_{4}$ & 74 & $160-62$ & $\mathrm{C}_{17} \mathrm{H}_{16} \mathrm{~N}_{4} \mathrm{O}_{2}$ & $66 \cdot 20$ & $5 \cdot 21$ & $18 \cdot 16$ \\
\hline & & & & $(308 \cdot 33)$ & $(66 \cdot 22$ & $5 \cdot 23$ & $18 \cdot 17)$ \\
\hline $4 m$ & $4-\mathrm{OCH}_{3} \mathrm{C}_{6} \mathrm{H}_{4}$ & 75 & $155-57$ & $\mathrm{C}_{17} \mathrm{H}_{16} \mathrm{~N}_{4} \mathrm{O}_{2}$ & $66 \cdot 19$ & $5 \cdot 20$ & $18 \cdot 14$ \\
\hline & & & & $(308 \cdot 33)$ & $(66 \cdot 22$ & $5 \cdot 23$ & $18 \cdot 17)$ \\
\hline $5 \mathbf{a}$ & $-\mathrm{CH}_{3}$ & 86 & $188-90$ & $\mathrm{C}_{13} \mathrm{H}_{13} \mathrm{ClN}_{4} \mathrm{O}_{2}$ & $53 \cdot 31$ & $4 \cdot 46$ & $19 \cdot 11$ \\
\hline & & & & $(292 \cdot 72)$ & $(53 \cdot 34$ & $4 \cdot 48$ & $19 \cdot 14)$ \\
\hline $5 \mathbf{b}$ & $-\mathrm{CH}_{2} \mathrm{CH}_{3}$ & 80 & $185-87$ & $\mathrm{C}_{14} \mathrm{H}_{15} \mathrm{ClN}_{4} \mathrm{O}_{2}$ & $54 \cdot 79$ & 4.92 & $18 \cdot 24$ \\
\hline & & & & $(306 \cdot 75)$ & $(54 \cdot 82$ & 4.93 & $18 \cdot 26)$ \\
\hline $5 c$ & $-\mathrm{CH}_{2} \mathrm{CH}_{2} \mathrm{CH}_{3}$ & 82 & $193-95$ & $\mathrm{C}_{15} \mathrm{H}_{17} \mathrm{ClN}_{4} \mathrm{O}_{2}$ & $56 \cdot 12$ & $5 \cdot 31$ & $17 \cdot 44$ \\
\hline & & & & $(320 \cdot 77)$ & $(56 \cdot 16$ & $5 \cdot 34$ & $17 \cdot 47)$ \\
\hline $5 d$ & $-\mathrm{C}_{6} \mathrm{H}_{5}$ & 86 & $150-52$ & $\mathrm{C}_{18} \mathrm{H}_{15} \mathrm{ClN}_{4} \mathrm{O}_{2}$ & $60 \cdot 92$ & $4 \cdot 24$ & $15 \cdot 76$ \\
\hline & & & & $(354 \cdot 79)$ & $(60 \cdot 94$ & $4 \cdot 26$ & $15 \cdot 79)$ \\
\hline $5 e$ & $2-\mathrm{CH}_{3} \mathrm{C}_{6} \mathrm{H}_{4}$ & 64 & $147-49$ & $\mathrm{C}_{19} \mathrm{H}_{17} \mathrm{ClN}_{4} \mathrm{O}_{2}$ & $61 \cdot 84$ & $4 \cdot 63$ & $15 \cdot 17$ \\
\hline & & & & $(368 \cdot 82)$ & $(61 \cdot 87$ & $4 \cdot 65$ & $15 \cdot 19)$ \\
\hline $5 f$ & $3-\mathrm{CH}_{3} \mathrm{C}_{6} \mathrm{H}_{4}$ & 79 & $149-51$ & $\mathrm{C}_{19} \mathrm{H}_{17} \mathrm{ClN}_{4} \mathrm{O}_{2}$ & 61.85 & $4 \cdot 62$ & $15 \cdot 16$ \\
\hline & & & & $(368 \cdot 82)$ & $(61 \cdot 87$ & $4 \cdot 65$ & $15 \cdot 19)$ \\
\hline $5 g$ & $2-\mathrm{ClC}_{6} \mathrm{H}_{4}$ & 75 & $141-43$ & $\mathrm{C}_{18} \mathrm{H}_{14} \mathrm{Cl}_{2} \mathrm{~N}_{4} \mathrm{O}_{2}$ & $55 \cdot 51$ & $3 \cdot 61$ & $14 \cdot 35$ \\
\hline & & & & $(389.24)$ & $(55 \cdot 54$ & 3.63 & $14 \cdot 39)$ \\
\hline $5 h$ & $4-\mathrm{ClC}_{6} \mathrm{H}_{4}$ & 84 & $160-62$ & $\mathrm{C}_{18} \mathrm{H}_{14} \mathrm{Cl}_{2} \mathrm{~N}_{4} \mathrm{O}_{2}$ & $55 \cdot 52$ & $3 \cdot 60$ & $14 \cdot 37$ \\
\hline & & & & $(389.24)$ & $(55 \cdot 54$ & $3 \cdot 63$ & 14.39) \\
\hline $5 \mathbf{i}$ & $2-\mathrm{OHC}_{6} \mathrm{H}_{4}$ & 85 & $120-22$ & $\mathrm{C}_{18} \mathrm{H}_{15} \mathrm{ClN}_{4} \mathrm{O}_{3}$ & $58 \cdot 29$ & $4 \cdot 07$ & $15 \cdot 09$ \\
\hline & & & & $(370 \cdot 79)$ & $(58 \cdot 31$ & $4 \cdot 08$ & $15 \cdot 11)$ \\
\hline $5 \mathbf{j}$ & $3-\mathrm{OHC}_{6} \mathrm{H}_{4}$ & 87 & $110-112$ & $\mathrm{C}_{18} \mathrm{H}_{15} \mathrm{ClN}_{4} \mathrm{O}_{3}$ & $58 \cdot 28$ & $4 \cdot 05$ & $15 \cdot 07$ \\
\hline & & & & $(370 \cdot 79)$ & $(58 \cdot 31$ & $4 \cdot 08$ & $15 \cdot 11)$ \\
\hline $5 \mathbf{k}$ & $4-\mathrm{OHC}_{6} \mathrm{H}_{4}$ & 84 & $126-28$ & $\mathrm{C}_{18} \mathrm{H}_{15} \mathrm{ClN}_{4} \mathrm{O}_{3}$ & $58 \cdot 26$ & $4 \cdot 05$ & $15 \cdot 08$ \\
\hline & & & & $(370 \cdot 79)$ & $(58 \cdot 31$ & $4 \cdot 08$ & $15 \cdot 11)$ \\
\hline 51 & $2-\mathrm{OCH}_{3} \mathrm{C}_{6} \mathrm{H}_{4}$ & 81 & $135-37$ & $\mathrm{C}_{19} \mathrm{H}_{17} \mathrm{ClN}_{4} \mathrm{O}_{3}$ & $59 \cdot 27$ & $4 \cdot 43$ & $14 \cdot 53$ \\
\hline & & & & $(384 \cdot 82)$ & $(59 \cdot 30$ & $4 \cdot 45$ & $14 \cdot 56)$ \\
\hline $5 \mathrm{~m}$ & $4-\mathrm{OCH}_{3} \mathrm{C}_{6} \mathrm{H}_{4}$ & 84 & $151-53$ & $\mathrm{C}_{19} \mathrm{H}_{17} \mathrm{ClN}_{4} \mathrm{O}_{3}$ & $59 \cdot 27$ & $4 \cdot 41$ & 14.53 \\
\hline & & & & $(384 \cdot 82)$ & $(59 \cdot 30$ & $4 \cdot 45$ & $14 \cdot 56)$ \\
\hline
\end{tabular}


Table 2. Antibacterial activity of compounds (5a-5m).

\begin{tabular}{|c|c|c|c|c|c|c|}
\hline \multirow[b]{3}{*}{ Compounds } & \multicolumn{6}{|c|}{ Mean zone inhibition (in $\mathrm{mm})^{\mathrm{a}}$} \\
\hline & \multicolumn{3}{|c|}{ Gram-positive bacteria } & \multicolumn{3}{|c|}{ Gram-negative bacteria } \\
\hline & S. aureus & S. mutans & B. subtilis & E. coli & S. typhi & P. aeruginosa \\
\hline $5 \mathbf{a}$ & 38 & 20 & 28 & 18 & 18 & 14 \\
\hline $5 \mathbf{b}$ & 37 & 18 & 28 & - & 16 & - \\
\hline $5 c$ & 32 & 18 & 22 & - & - & 12 \\
\hline 5d & 36 & 16 & 27 & - & 13 & 12 \\
\hline $5 e$ & 30 & 15 & 20 & - & - & - \\
\hline $5 \mathbf{f}$ & 30 & 13 & 20 & - & - & - \\
\hline $5 g$ & 31 & 13 & 18 & - & - & - \\
\hline $5 \mathrm{~h}$ & 31 & 10 & 20 & - & - & - \\
\hline $5 i$ & 37 & 16 & 27 & - & 10 & 10 \\
\hline $5 \mathbf{j}$ & 36 & 16 & 26 & - & 10 & - \\
\hline $5 \mathbf{k}$ & 36 & 15 & 26 & - & - & - \\
\hline 51 & 25 & 14 & 22 & - & - & - \\
\hline $5 \mathrm{~m}$ & 22 & 16 & 18 & - & - & - \\
\hline Ampicillin $^{\mathrm{b}}$ & 38 & 22 & 28 & 20 & - & - \\
\hline Nalidixic acid & - & - & - & 28 & 20 & 18 \\
\hline
\end{tabular}

\footnotetext{
${ }^{\mathrm{a}}$ Values are mean $(n=3)$

${ }^{\mathrm{b}}$ Ampicillin (10 $\left.\mu \mathrm{g} / \mathrm{disc}\right)$ and Nalidixic acid (30 $\left.\mu \mathrm{g} / \mathrm{disc}\right)$ used as positive reference; synthesized compounds $(300 \mu \mathrm{g} / \mathrm{disc})$

'-' indicates no sensitivity or mean inhibition zone diameter lower than $7 \mathrm{~mm}$
}

Table 3. Antifungal activity of compounds (5a-5m).

\begin{tabular}{lccc}
\hline & \multicolumn{3}{c}{ Mean zone inhibition (in mm) } \\
Compounds & Candida albicans & Aspergillus niger & Aspergillus flavus \\
\cline { 2 - 4 } 5a & 26 & 24 & 22 \\
$\mathbf{5 b}$ & 24 & 25 & 24 \\
$\mathbf{5 c}$ & 24 & 24 & 20 \\
$\mathbf{5 d}$ & 27 & 26 & 27 \\
$\mathbf{5 e}$ & 22 & 18 & 14 \\
$\mathbf{5 f}$ & 20 & 18 & 13 \\
$\mathbf{5 g}$ & 20 & 16 & 15 \\
$\mathbf{5 h}$ & 18 & 14 & 14 \\
$\mathbf{5 i}$ & 27 & 28 & 26 \\
$\mathbf{5 j}$ & 15 & 20 & 13 \\
$\mathbf{5 k}$ & 15 & 14 & 18 \\
$\mathbf{5 l}$ & 16 & 16 & 16 \\
$\mathbf{5 m}$ & 18 & 19 & 16 \\
${ }^{\mathrm{b}}$ Amphotericin B & 28 & $>28$ & 28 \\
\hline
\end{tabular}

${ }^{a}$ Values are mean $(n=3)$

${ }^{\mathrm{b}}$ Amphotericin B (30 $\left.\mu \mathrm{g} / \mathrm{disc}\right)$ used as positive reference; synthesized compounds $(300 \mu \mathrm{g} / \mathrm{disc})$

The results of the final compounds for preliminary antibacterial testing are shown in table 2 . The results revealed that the majority of the synthesized compounds showed varying degree of inhibition against the tested microorganisms. In general, the inhibitory activity against the Gram-positive bacteria was higher than that of the Gram-negative bacteria. The alkyl, phenyl and hydroxyphenyl substitutions at the 4-position of azetidin-2-one subunit has the best overall antibacterial profile. The methyl, chloro 


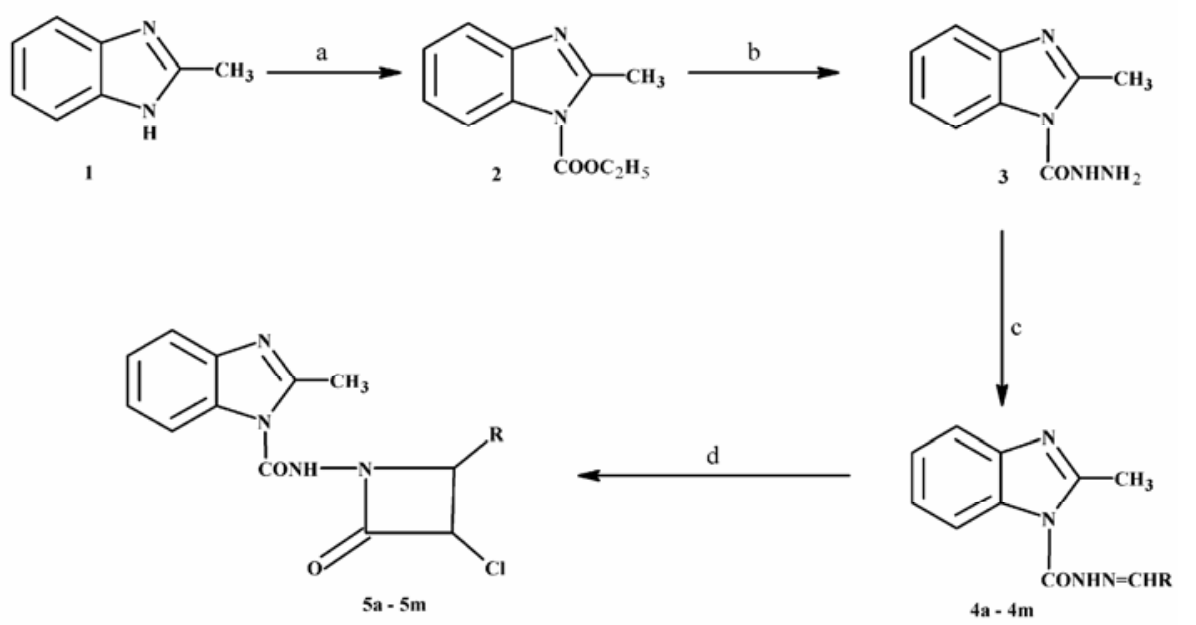

Scheme 1. (Reagents: (a) $\mathrm{ClCOOC}_{2} \mathrm{H}_{5} / \mathrm{K}_{2} \mathrm{CO}_{3}$, acetone; (b) $\mathrm{NH}_{2} \mathrm{NH}_{2} \cdot \mathrm{H}_{2} \mathrm{O}$ / ethanol; (c) corresponding aldehyde/ethanol (d) $\mathrm{ClCOCH}_{2} \mathrm{Cl} / \mathrm{Et}_{3} \mathrm{~N}$ ).

and methoxy substituents on phenyl ring at azetidin2-one moiety of final compounds displayed least activity.

As can be seen in table 3, although all the compounds are not as active as standard Amphotericin B, compounds $\mathbf{5 d}$ and $\mathbf{5 i}$ were found to be more active against Candida albicans and Aspergillus flavus. Again in antifungal activity compounds 5e, $\mathbf{5 f}, \mathbf{5 g}, \mathbf{5 h}, \mathbf{5 l}$ and $\mathbf{5 m}$ showed less or negligible activity than the other derivatives of the same series. Although the rest of the compounds showed varying degree of inhibition, none were as effective as Amphotericin B.

\section{Conclusions}

We have synthesized series of $N$-[3-chloro-2(substituted) alkyl/aryl-4-oxoazetidin-1-yl]-1-carboxamido-2-methyl- $1 H$-benzimidazoles. Among the synthesized benzimidazoles, compounds with alkyl, phenyl and hydroxyphenyl at 4-position of azetidin2-one were found to increase the antibacterial activity. Compounds with phenyl and hydroxyphenyl substituents at azetidin-2-one sub-unit showed good antibacterial and antifungal activities. More extensive study is needed to confirm the preliminary results and mode of action studies are required to be able to optimize the effectiveness of this series of compounds.

\section{References}

1. (a) Wright J B 1951 Chem. Rev. 48 397; (b) Preston P N 1974 Chem. Rev. 74279
2. Preston P N 1980 In Benzimidazole and congeneric tricyclic compounds (eds) A Weissberger and E C Taylor (New York: John Wiley and Sons), Part-2, pp. 63-147; (b) Grimmett M R 1984 In Comprehensive heterocyclic chemistry (ed.) K T Pots (Oxford: Pergamon) pp. 345-498; (c) Hoffman K 1953 In Imidazole and its derivatives (ed.) A Weissberger; The chemistry of heterocyclic compounds (New York: Interscience Publishers, Inc) pp. 247-317

3. Kus C, Ayhan-Kilcigil G, Can Eke B and Iscan M 2004 Arch. Pharm. Res. 27156

4. Ates-Alagoz Z, Kus C and Coban T 2005 J. Enzyme Inhib. Med. Chem. 20325

5. Goker H, Kus C, Boykin D W, Yildiz S and Altanlar N 2002 Bioorg. Med. Chem. 102589

6. Goker H, Ozden S, Yildiz S and Boykin D W 2005 Eur. J. Med. Chem. 401062

7. Desai K G and Desai K R 2006 Bioorg. Med. Chem. 148271

8. Kazimierczuk Z, Upcroft J A, Upcroft P, Gorska A, Starosciak B and Laudy A 2002 Acta Biochim. Polon. 49185

9. Mohammad B G, Hussien M A, Abdel-Alim A A and Hashem M 2006 Arch. Pharm. Res. 2926

10. Pawar N S, Dalal D S, Shimpi S R and Mahulikar P P 2004 Eur. J. Pharm. Sci. 21115

11. Dubey R, Abuzar S, Sharma S, Chatterjee R K and Katiyar J C 1985 J. Med. Chem. 281748

12. Mavrova A T, Denkova P S, Tsenov Y A, Anichina K K and Vutchev D L 2007 Bioorg. Med. Chem. 15 6291

13. Ravina E, Sanchez-Alonso R, Fueyo J, Baltar M P, Bos J, Iglesias R and Sanmartin M L 1993 Arzneim. Forsch. 43684

14. Starcevic K, Kralj M, Ester K, Sabol I, Grce M, Pavelic K and Karminski-Zamola G 2007 Bioorg. Med. Chem. 154419

15. Kubo K, Inada Y, Kohara Y, Sugiura Y, Ojima M, Itoh K, Furukawa Y, Nishikawa Y K and Naka T 1993 J. Med. Chem. 361772 
16. Ram S, Wise D S, Wotring L L, McCall J W and Townsend L B 1992 J. Med. Chem. 35539

17. Lazer E S, Matteo M R and Possanza G J $1987 \mathrm{~J}$. Med. Chem. 30726

18. Lackner T E and Clissold S P 1989 Drugs 38204

19. Ito K, Kagaya H, Fukuda T, Yoshino K and Nose $T$ 1982 Arzneim. Forsch. Drug Res. 3249

20. Navarette-Vazquez G, Cedilla R, Hernandez-Campos A, Yepez A, Hernandez-Luis F, Valdez J, Morales R, Cortes R, Hernandez M and Castillo R 2001 Bioorg. Med. Chem. 11187

21. Katiyar S K, Gordon V R, McLaughlin G L and Edlind T D 1994 Antimicrob. Agents Chemother. 382986

22. Li Y F, Wang G F, He P L, Huang W G, Zhu F H, Gao H Y, Tang W, Luo Y, Feng C L, Shi L P, Ren Y D, Lu W and Zuo J P 2006 J. Med. Chem. 494790

23. Kidwai M, Sapra P and Bhushan K R 1999 Curr. Med. Chem. 6195

24. Deshmukh A R, Bhawal B M, Krishnaswamy D, Govande V V, Shinkre B A and Jayanthi A 2004 Curr. Med. Chem. 111889
25. Diurno M V, Mazzoni O, Piscopo E and Bolognese A 1992 Il Farmaco 47239

26. Alcaide B and Almendros P 2004 Curr. Med. Chem. 111921

27. Singh S G and Boycie J M 2005 Il Farmaco 60727

28. Vaccaro W D, Sher R and Jr Davis H R 1998 Bioorg. Med. Chem. Lett. 8319

29. Ansari K F and Lal C 2009 Eur. J. Med. Chem. 44 4028

30. Ansari K F and Lal C 2009 Eur. J. Med. Chem. 44 2294

31. National Committee for Clinical Laboratory Standards, Performance Standards for Antimicrobial Disk Susceptibility tests; Approved Standard M2-A8, Clinical and Laboratory Standards Institute, Wayne, PA, USA, 2003

32. (a) Furniss B S, Hannaford A J, Smith P W G and Tatchell A R 1998 Vogel's text book of practical organic chemistry (England: ELBS Longman) pp. 1162-1163; (b) Registry No. 301684-45-7 (for compound 2) 